\title{
Confiabilidade da classificação do estado nutricional obtida através do IMC e três diferentes métodos de percentual de gordura corporal em pacientes com diabetes melito tipo 1
}

\author{
Reliability of the classification of nutritional status obtained \\ through the BMI and three different methods of body fat \\ percentage in patients with type 1 diabetes mellitus
}

Raquel Ramalho Nunes', Eliete Leão da Silva Clemente', Juliana Almeida Pandini', Roberta Arnoldi Cobas'

Viviane Monteiro Dias' ${ }^{1}$, Sandro Sperandei ${ }^{2}$, Marília de Brito Gomes ${ }^{1}$

\section{RESUMO}

Objetivo: Avaliar a confiabilidade da classificação do estado nutricional (EN) obtida através do índice de massa corporal (IMC) e três diferentes métodos de composição corporal (CC) em indivíduos diabéticos tipo 1 (DM1) e não diabéticos. Métodos: Foram avaliados 84 pacientes com DM1 e 37 controles. Coletaram-se os dados antropométricos para calcular o IMC e a avaliação

${ }^{1}$ Departamento de Medicina, Disciplina de Diabetes e Metabologia, Hospital Universitário Pedro Ernesto, Universidade do Estado do Rio de Janeiro (Ueri), Rio de Janeiro, RJ, Brasil ${ }^{2}$ Centro de Desenvolvimento Tecnológico em Saúde, Fundação Oswaldo Cruz (Fiocruz), Rio de Janeiro, RJ, Brasil
Correspondência para: Raquel Ramalho Nunes Hospital Universitário Pedro Ernesto, Disciplina de Diabetes e Metabologia

Av. 28 de Setembro, 77, 3andar 20551-030 - Rio de Janeiro, RJ, Brasil

raquelrnunes@hotmail.com

Recebido em 6/Ago/2008 Aceito em 29/Nov/2008 da CC foi obtida por meio dos métodos de dobras cutâneas (DC), bioimpedância elétrica bipolar (BI) e tetrapolar (TT). A adequação entre as classificações de cada método foi determinada pelo coeficiente Kappa (K). Resultados: Dentre os 84 pacientes, apenas $48(57,1 \%)$ apresentaram classificação do IMC concordante com o método de DC, $58(69 \%)$ com o de BI e $45(53,5 \%)$ com o deTT. Os resultados do $\mathrm{K}$ para os indivíduos com $\mathrm{DM} 1$ foi de $\mathrm{DC}=0,261, \mathrm{BI}=0,320$ eTT =0,174. Os controles apresentaram valores maiores $(\mathrm{DC}=0,605, \mathrm{BI}=0,360$ eTT $=0,400)$. Porém, todos os valores foram considerados baixos. Conclusões: 0 método de IMC mostrou-se pouco sensível às variações na $\mathrm{CC}$ dos indivíduos com DM1. Métodos próprios para a avaliação da CC devem ser utilizados na classificação do EN dessa população. Arq Bras Endocrinol Metab. 2009;53(3):360-7.

Descritores

Diabetes melito tipo 1; índice de massa corporal; composição corporal; percentual de gordura corporal

\section{ABSTRACT}

Objective: To assess the reliability of classification of nutritional status (NS) obtained through the body mass index (BMI) and three different methods of body composition (BC) in individuals type 1 diabetics (T1D) and non-diabetic subjects. Methods: 84 patients with T1D and 37 controls were evaluated. Anthropometric data was collected to calculate BMI and assessment of BC was performed through the methods of skinfold thickness (SF), bipolar (BI) and tetrapolar (TT) bioelectrical impedance. The agreement between the scores of each method was determined by Kappa (K) coefficient. Results: Considering all the patients, only $48(57.1 \%)$ presented classification of BMI that agreed with the SF method, $58(69 \%)$ with the BI and $45(53.5 \%)$ with the TT. The $\mathrm{K}$ results for individuals withT1D was $\mathrm{DC}=0.261, \mathrm{BI}=0.320$ and $T \mathrm{~T}=0.174$. The controls had higher values $(\mathrm{DC}=0.605, \mathrm{BI}=0.360$ and $\Pi T=0.400$ ). However, all values were considered low. Conclusions: The method of BMI showed little sensitivity to $\mathrm{BC}$ changes in patients withT1D. Appropriated methods for the assessment of BC should be used to classify the NS of this population. Arq Bras Endocrinol Metab. 2009;53(3):360-7.

Keywords

Type 1 diabetes mellitus; body mass index; body composition; body fat percentage 


\section{INTRODUÇÃO}

$A$ p prevalência de diabetes melito $(\mathrm{DM})$, sobrepeso e obesidade vem aumentando de forma progressiva nos últimos anos, tanto nos países desenvolvidos como nos países em desenvolvimento (1-4).

Indivíduos com diabetes melito tipo l (DMl) apresentavam, como característica clínica no início da doença, baixo peso e/ou peso normal. A ocorrência de sobrepeso ou obesidade nesses pacientes durante a evolução da doença era, na maioria das vezes, um fato raro e isolado (5-7). Esses dois conceitos acima descritos vêm apresentando uma mudança gradativa nos últimos anos, consequente ao aumento significativo da prevalência de sobrepeso e obesidade em adultos, crianças e adolescentes na população mundial (8-12).

Pacientes com DMl têm risco duas a quatro vezes maior de desenvolver aterosclerose em relação à população não diabética (13). Torna-se importante identificar a presença de fatores de risco para doença cardiovascular nessa população. $\mathrm{O}$ principal fator de risco para desenvolvimento de dislipidemia na infầncia, avaliados em uma metanálise de 33 estudos, foi sobrepeso ou composição corporal de gordura (14).

O índice de massa corporal (IMC) é o método mais utilizado para classificação de sobrepeso ou obesidade nas populações adultas (15). É um método simples, prático, rápido, de fácil aplicabilidade e mensuração, além de requerer menos treinamento e equipamentos mais baratos (16-19). O IMC pode até ser calculado a partir de valores relatados pelo avaliado, dispensando a necessidade de aferição sem prejuízo significativo para classificação do estado nutricional (20-25). Dessa forma, o IMC apresenta grande vantagem em relação aos métodos de avaliação da composição corporal, sendo então o mais escolhido na rotina clínica e em estudo epidemiológico (26).

No entanto, quando comparado a métodos de avaliação da composição corporal, o IMC demonstra uma estimativa imprecisa da massa de gordura e da massa magra (massa livre de gordura) e não fornece informação a respeito de as alterações de peso resultarem de decréscimo ou aumento de massa magra e/ou massa de gordura (27). Alguns autores já demonstraram que ele não reflete a real composição corporal dos indivíduos, questionando-se o seu uso com o propósito de diagnosticar a gordura corporal (28-32).

Métodos de avaliação da composição corporal mediante análise compartimentalizada do peso corporal total permitem determinar as diversas porções que cada tecido corporal ocupa no organismo, podendo oferecer resultados mais precisos a respeito de cada um deles (29). Alguns desses métodos já têm sido utilizados como rotina clínica e, até mesmo, em estudos de pesquisa epidemiológica de larga escala (34-36).

Estão disponíveis como métodos para análise da composição corporal, por meio da estimativa do percentual de gordura corporal, técnicas mais complexas e sofisticadas que requerem equipamentos de alto custo e não portáteis, como a hidrodensitometria, a diluição de isótopos e a absortiometria de raios $\mathrm{X}$ de dupla energia (DEXA), e técnicas relativamente simples e baratas, como as que empregam os valores de dobras cutâneas e a bioimpedância elétrica (BIA) $(33,37)$.

A medida do percentual de gordura corporal obtido pela mensuração de dobras cutâneas tem tido larga aceitação entre os pesquisadores, já que por intermédio dessa técnica antropométrica os valores de percentual de gordura corporal associam-se muito bem e não diferem significativamente dos valores decorrentes da pesagem hidrostática (padrão-ouro) (38-43).

Quanto à BIA, os dados fornecidos por essa técnica não constituem medidas diretas de qualquer estrutura biológica de interesse como, por exemplo, gordura ou massa muscular. Nessa técnica, as estimativas são obtidas a partir de equações empíricas que fornecem valores para a água total do organismo e, então, o teor de massa magra e o teor de gordura, num modelo de dois compartimentos. Assim, no que concerne à composição do corpo avaliada pela BIA, inúmeras equações foram desenvolvidas, considerando-se sexo, idade, estatura e, também, doenças (44-46).

Diferentes métodos de avaliação da composição corporal foram comparados em indivíduos saudáveis (47-52) e em indivíduos acometidos por enfermidades (53-55). Entretanto, estudos em populações de indivíduos diabéticos tipo 1 ainda não são numerosos e conclusivos na literatura (13,56-59). Esse fato confirma a necessidade de estudos nessa população nos quais técnicas simples e de fácil aplicabilidade possam ser utilizadas, tornando possível a determinação da quantidade de tecido adiposo de cada indivíduo e sua correlação com o diabetes e suas complicações.

\section{OBJETIVO}

O objetivo do presente estudo foi avaliar a confiabilidade da classificação do estado nutricional obtida por meio do IMC e três diferentes métodos de percentual 
de gordura corporal em indivíduos diabéticos tipo 1 comparados a um grupo de não diabéticos.

\section{METODOLOGIA}

\section{Indivíduos}

Foram estudados 84 indivíduos com DMl ( $54 \% \mathrm{~F} / 46 \%$ M) com idade média de 31,6 $\pm(10,58)$ anos, $14,5 \pm$ $(8,01)$ anos de duração do diabetes, utilizando $0,8 \pm$ $(0,31) \mathrm{U} / \mathrm{kg} /$ dia de insulina que foram acompanhados pela mesma equipe no Ambulatório de Diabetes do Hospital Universitário Pedro Ernesto (HUPE) da Universidade do Estado do Rio de Janeiro (UERJ), pareados por idade, sexo e IMC com 37 indivíduos não diabéticos $(49 \%$ F/51\% M) e com idade média de 28,1 $\pm(7,3)$ anos.

\section{Materiais}

Para aferição do peso, foi utilizada uma balança antropométrica da marca Filizola com capacidade para $150 \mathrm{~kg}$. Para estatura, um estadiômetro de parede, marca Tonelli \& Gomes. Para BIA tetrapolar, o aparelho da marca Quantum RJL Systems. Para as dobras cutâneas, um adipômetro Lange da marca Cambrige Scientific Industries com precisão de $0,2 \mathrm{~mm}$. E, para BIA bipolar, o aparelho da marca OMRON, Modelo HBF-306BL. A coleta de dados foi realizada em ficha própria elaborada pelo examinador. Os dados foram tabulados em planilha de cálculo elaborada pelo autor, por meio do software Microsoft ${ }^{\circledR}$ Excel (EUA).

\section{Métodos}

Todos os indivíduos envolvidos no estudo foram orientados a virem à consulta na data solicitada em jejum de 12 horas. No dia e horário marcados, antes de dar início à avaliação da composição corporal, eram explicados os procedimentos, sendo entregue a cada voluntário o termo de consentimento livre e informado para assinarem, liberando a sua participação no estudo. Só depois eram submetidos à entrevista a partir da qual eram coletados dados pessoais e realizadas as medidas da composição corporal.

A aferição dos parâmetros da composição corporal foi realizada na seguinte ordem: (1) peso, (2) estatura, (3) BIA tetrapolar, (4) dobras cutâneas (tríceps, suprailíaca, coxa em mulheres; peitoral, abdominal e coxa em homens e subescapular em ambos os sexos) e (5) BIA bipolar. As aferições de resistência e reactância feitas no aparelho de BIA tetrapolar foram realizadas mediante colocação de quatro eletrodos no corpo do indivíduo avaliado, posicionados em locais predeterminados pelas instruções do aparelho. Já a medição feita no aparelho de BIA bipolar foi realizada apenas pelo contato das mãos com os dois eletrodos acoplados diretamente ao aparelho.

Todos os critérios utilizados para medição dos parâmetros necessários à avaliação da composição corporal seguiram os protocolos descritos por Guedes e Guedes (61) e Heyward e Stolarczyk (62).

Todas as medições foram realizadas três vezes pelo mesmo examinador, sendo considerada a média aritmética das três medidas ao final. Posteriormente, foram calculados o IMC e o percentual de gordura corporal.

Todas as aferições realizadas para avaliação da composição corporal seguiram um protocolo específico. Para a BIA: (1) não comer ou beber quatro horas antes do exame, (2) não fazer exercícios 12 horas antes do exame, (3) urinar 30 minutos antes do exame, (4) não consumir álcool 48 horas antes do exame, (5) não fazer uso de diuréticos sete dias antes do exame e (6) não estar no período menstrual. E, para as dobras cutâneas: (1) aferir as medições em posição ortostática e em repouso, sempre no hemicorpo direito, (2) identificar e marcar cuidadosamente o ponto anatômico a ser medido, (3) pinçar com o polegar e o indicador a dobra de pele e gordura afastando o tecido muscular subjacente, (4) aplicar a borda superior do compasso perpendicular a dobra a cerca de $1 \mathrm{~cm}$ abaixo do ponto exato de reparo, (5) soltar a pressão das hastes do compasso sem soltar a dobra dos dedos e (6) aguardar por volta de quatro segundos após soltar a pressão das hastes para que a leitura do resultado seja feita.

Para classificação do peso, foram utilizados os critérios de IMC propostos pela World Health Organization (WHO) (2). O mesmo foi calculado dividindo-se o peso $(\mathrm{kg})$ pela altura ao quadrado $\left(\mathrm{m}^{2}\right)$. Sendo os indivíduos adultos de ambos os sexos e com idade igual ou superior a 18 anos classificados da seguinte forma: baixo peso (IMC $<18,5 \mathrm{~kg} / \mathrm{m}^{2}$ ), normal (IMC entre $18,5 \mathrm{e}$ $24,9 \mathrm{~kg} / \mathrm{m}^{2}$ ), sobrepeso (IMC entre 25 e $29,9 \mathrm{~kg} / \mathrm{m}^{2}$ ) e obesidade (IMC $\geq 30 \mathrm{~kg} / \mathrm{m}^{2}$ ).

Para determinação do percentual de gordura corporal pelo método de BIA tetrapolar, foi utilizado um programa computacional específico (Comp Corp Sistem - Programa de avaliação corporal por bioimpedância - Versão 2.5). Para o método de dobras cutâneas, foi utilizado o protocolo de três dobras, sendo a equação preditiva do percentual de gordura de Jackson e Pollo- 
ck (38) utilizada para os indivíduos do sexo masculino com idade entre 18 a 61 anos e a de Jackson e cols. (39) para os indivíduos do sexo feminino com idade entre 18 a 55 anos. E, para o método de BIA bipolar, o resultado é mostrado diretamente no próprio aparelho. A classificação do percentual de gordura corporal foi dada mediante critério de Gallagher e cols. (62).

Este estudo foi aprovado pelo Comitê de Ética em Pesquisa do HUPE.

\section{Análise estatística}

Os dois grupos foram comparados nas variáveis: peso, estatura, IMC e percentual de gordura nos três métodos utilizados pelo teste $t$ de Student para amostras independentes com correção de Sidak para comparações múltiplas.

A adequação entre as classificações de cada método foi avaliada por meio do coeficiente Kappa de Cohen (63), onde $a$ representa o número de concordâncias entre os dois métodos em cada categoria, efé a frequência esperada para a concordância e $N$ é o total de indivíduos avaliados.

Os dados estão apresentados como média ( \pm desviopadrão). O nível de significância (valor de $\mathrm{p}$ ) foi estabelecido em 0,05 .

\section{RESULTADOS}

As características clínicas e demográficas dos pacientes e controles encontram-se descritas na tabela 1 . Não foram observadas diferenças significativas entre os dois grupos nas variáveis analisadas.

O resultado da classificação dos indivíduos diabéticos tipo 1 e controles por cada um dos métodos de percentual de gordura corporal em relação a uma das quatro categorias de IMC está descrito nas tabelas 2 e 3, assim como o valor do coeficiente Kappa para a comparação entre o IMC e o respectivo método de percentual de gordura corporal.

Os maiores erros apresentados entre as categorias se concentraram na faixa de peso normal (categoria 2) e sobrepeso (categoria 3) em ambos os grupos.

Na tabela 2, verifica-se que, entre os indivíduos diabéticos tipo 1, $48(57,1 \%), 58(69 \%)$ e $45(53,5 \%)$ foram classificados como baixo peso, peso normal, sobrepeso e obesidade pelos métodos de IMC e percentual de gordura corporal por dobras cutâneas, BIA bipolar e tetrapolar, respectivamente. Na tabela 3 verifica-se, que entre os indivíduos do grupo controle, 28 (75,6\%), 23 $(62,1 \%)$ e $22(59,4 \%)$ foram classificados como baixo peso, peso normal, sobrepeso e obeso pelos métodos de IMC e percentual de gordura corporal por dobras cutâneas, BIA bipolar e tetrapolar, respectivamente.

Observou-se que os resultados apresentados pelo coeficiente Kappa no grupo controle de 0,605 para re-

\begin{tabular}{lccc}
\hline \multicolumn{4}{c}{ Tabela 1. Dados descritivos das amostras selecionadas } \\
\hline & Diabéticos & Controles & Valor de p \\
\hline n total & 84 & 37 & \\
n por sexo (F/M) & $45 / 39$ & $18 / 19$ \\
Duração do diabetes (anos) & $14,5(8,01)$ & - & \\
Dose de insulina (U/kg/dia) & $0,8(0,31)$ & - & \\
Idade (anos) & $31,6(10,58)$ & $28,1(7,3)$ & 0,4 \\
Massa corporal (kg) & $65,5(11,36)$ & $67,3(15,01)$ & 0,1 \\
Estatura (m) & $1,7(0,09)$ & $1,7(0,10)$ & 0,1 \\
IMC (kg/m²) & $23,6(3,23)$ & $23,6(4,38)$ & 0,1 \\
\% GC DC & $24,9(9,85)$ & $25,0(8,9)$ & 0,1 \\
\% GC BIA BI & $21,4(7,68)$ & $21,5(7,05)$ & 0,1 \\
\% GC BIA TT & $26,2(6,93)$ & $28,4(5,93)$ & 0,9 \\
\hline
\end{tabular}

Valores expressos em média ( \pm desvio padrão).

\%GC DC: percentual de gordura corporal por dobras cutâneas; \%GC BIA BI: percentual de gordura corporal por bioimpedância elétrica bipolar; \%GC TT: percentual de gordura corporal por bioimpedância tetrapolar.

\begin{tabular}{|c|c|c|c|c|c|c|c|c|c|c|c|c|c|c|c|c|}
\hline & & \multicolumn{5}{|c|}{ \%GC DC } & \multicolumn{5}{|c|}{ \%GC BIA BI } & \multicolumn{5}{|c|}{ \%GC BIA TT } \\
\hline & & 1 & 2 & 3 & 4 & Total & 1 & 2 & 3 & 4 & Total & 1 & 2 & 3 & 4 & Total \\
\hline \multirow[t]{5}{*}{ IMC } & 1 & 2 & 0 & 0 & 0 & 2 & 2 & 0 & 0 & 0 & 2 & 0 & 2 & 0 & 0 & 2 \\
\hline & 2 & 9 & 37 & 8 & 4 & 58 & 9 & 48 & 1 & 0 & 58 & 4 & 36 & 15 & 3 & 58 \\
\hline & 3 & 0 & 7 & 7 & 6 & 20 & 0 & 14 & 6 & 0 & 20 & 0 & 6 & 7 & 7 & 20 \\
\hline & 4 & 0 & 0 & 2 & 2 & 4 & 0 & 1 & 1 & 2 & 4 & 0 & 0 & 2 & 2 & 4 \\
\hline & Total & 11 & 44 & 17 & 12 & 84 & 11 & 63 & 8 & 2 & 84 & 4 & 44 & 24 & 12 & 84 \\
\hline K & & \multicolumn{5}{|c|}{0,261} & \multicolumn{5}{|c|}{0,320} & \multicolumn{5}{|c|}{0,174} \\
\hline
\end{tabular}

\%GC DC: percentual de gordura corporal por dobras cutâneas; \%GC BIA BI: percentual de gordura corporal por bioimpedância elétrica bipolar; \%GC TT: percentual de gordura corporal por bioimpedância elétrica tetrapolar; (1) baixo peso; (2) normal; (3) sobrepeso; (4) obesidade; K: Kappa.

* Em negrito os valores que apresentaram concordância concomitante entre os métodos. 


\begin{tabular}{|c|c|c|c|c|c|c|c|c|c|c|c|c|c|c|c|c|}
\hline & & \multicolumn{5}{|c|}{ \%GC DC } & \multicolumn{5}{|c|}{$\%$ GC BIA BI } & \multicolumn{5}{|c|}{ \%GC BIA TT } \\
\hline & & 1 & 2 & 3 & 4 & Total & 1 & 2 & 3 & 4 & Total & 1 & 2 & 3 & 4 & Total \\
\hline \multirow[t]{5}{*}{ IMC } & 1 & 1 & 1 & 0 & 0 & 2 & 1 & 0 & 0 & 0 & 2 & 0 & 2 & 0 & 0 & 2 \\
\hline & 2 & 0 & 17 & 3 & 0 & 20 & 3 & 16 & 1 & 0 & 20 & 0 & 13 & 3 & 4 & 20 \\
\hline & 3 & 0 & 1 & 7 & 3 & 11 & 0 & 7 & 3 & 1 & 11 & 0 & 0 & 5 & 6 & 11 \\
\hline & 4 & 0 & 0 & 1 & 3 & 4 & 0 & 0 & 1 & 3 & 4 & 0 & 0 & 0 & 4 & 4 \\
\hline & Total & 1 & 19 & 11 & 6 & 37 & 4 & 24 & 5 & 4 & 37 & 0 & 15 & 8 & 14 & 37 \\
\hline \multicolumn{2}{|c|}{ K } & \multicolumn{5}{|c|}{0,605} & \multicolumn{5}{|c|}{0,360} & \multicolumn{5}{|c|}{0,400} \\
\hline
\end{tabular}

\%GC DC: percentual de gordura corporal por dobras cutâneas; \%GC BIA BI: percentual de gordura corporal por bioimpedância elétrica bipolar; \%GC TT: percentual de gordura corporal por bioimpedância elétrica tetrapolar; (1) baixo peso; (2) normal; (3) sobrepeso; (4) obesidade; K: Kappa.

* Em negrito os valores que apresentaram concordância concomitante entre os métodos.

lação entre IMC e percentual de gordura corporal por dobras cutâneas, 0,360 para relação entre IMC e percentual de gordura corporal por BIA bipolar e 0,400 para relação entre IMC e percentual de gordura corporal por BIA tetrapolar, embora tenham sido baixos, foram sempre maiores que os valores observados no grupo de indivíduos diabéticos tipo $1(0,261 ; 0,320 \mathrm{e}$ 0,174 para a relação entre IMC e percentual de gordura corporal por dobras cutâneas, BIA bipolar e tetrapolar, respectivamente).

\section{DISCUSSÃO}

A avaliação do estado nutricional de adultos requer o conhecimento da reserva energética e da massa metabolicamente ativa dos indivíduos, o que se obtém por meio da avaliação da composição corporal (36).

Tanto os indivíduos diabéticos tipo 1 quanto os indivíduos do grupo controle apresentaram resultados de percentual de gordura corporal semelhantes aos publicados na literatura $(56,59,62)$.

Embora o IMC seja um método internacionalmente aceito para classificação do estado nutricional, ele não avalia a composição corporal. O IMC é simplesmente uma relação entre o peso e a altura dos indivíduos, que apresenta limitações como: 1) a relação com a proporcionalidade do corpo - pessoas com pernas curtas terão IMC aumentado; 2) relação com a massa livre de gordura, especialmente em homens, pois um desenvolvimento muscular pode levar a interpretações equivocadas na identificação da obesidade; 3 ) correlação com a estatura que, apesar de baixa, pode ser significativa, especialmente em crianças e adolescentes (64) e, em idosos, a sarcopenia (redução da massa muscular), o acúmulo e a distribuição centrípeta da gordura são colocados como fatores que também limitam a utilização do $\operatorname{IMC}(30,65)$.
Os dados obtidos neste estudo ratificam os já descritos em outros estudos publicados na literatura $(29,66-68)$ que também demonstraram limitações do IMC $(69,70)$ nos indivíduos que apresentam um IMC dentro do padrão de normalidade, mas possuem uma quantidade de gordura corporal acima do ideal, confirmando relatos de que o IMC não é um bom marcador quantitativo da gordura corporal, podendo subestimar o percentual de gordura e classificar de forma errônea indivíduos com excesso de gordura (70). Outra limitação é o fato de indivíduos apresentando IMC abaixo do recomendado possivelmente possuírem quantidade de gordura corporal ideal. Essa falta de congruência entre o IMC e a gordura corporal pode ser explicada não só pela fragilidade desse índice, mas também pelo fato de a gordura corporal estar associada aos níveis de atividade ou aptidão física (71).

A adoção do IMC como padrão único de classificação do estado nutricional pode ocasionar avaliações imprecisas e um diagnóstico incorreto e, consequentemente, possíveis intervenções errôneas no tratamento tanto do diabetes como do excesso de peso. Esse fato foi observado em nosso estudo em que somente $28,5 \%$ e $40,5 \%$ dos indivíduos diabéticos tipo 1 e indivíduos do grupo controle, respectivamente, apresentaram IMC acima do recomendado. Porém, ao se verificarem os valores de percentual de gordura corporal acima do ideal encontrados nos indivíduos diabéticos tipo 1 e indivíduos normais, observou-se um aumento significativo desse número, confirmando evidências obtidas na literatura que indicam uma prevalência bem maior de pessoas com sobrepeso e obesidade $(9,72)$.

Nossos resultados demonstraram que no grupo de diabéticos tipo 1 houve uma concordância especialmente baixa entre os valores observados para classificação do estado nutricional pelo método de IMC e os valores encontrados para classificação do percentual de gordura 
corporal, demonstrando que a utilização do IMC como método de classificação do estado nutricional em diabéticos tipo 1 não é o mais adequado.

Independentemente do método utilizado, a concordância entre a classificação pelo método de IMC e os métodos de percentual de gordura corporal foi sempre melhor no grupo de indivíduos controles que no grupo de diabéticos tipo 1. Nesse mesmo grupo, a concordância foi maior entre a classificação pelo método de IMC e o método de BIA bipolar. Já no grupo controle, houve uma concordância maior entre a classificação pelo método de IMC e o método de dobras cutâneas. Entretanto, isso não significa que se deva utilizar o método de BIA bipolar no grupo específico, uma vez que o valor da concordância ainda foi baixo.

Em estudos nos quais a concordância entre os métodos de avaliação do percentual de gordura corporal também foi avaliada, Freitas Júnior e cols. (53) observaram que, em pacientes com doença pulmonar obstrutiva crônica, os valores de percentual de gordura corporal apresentaram diferença significativa quando comparados à BIA e ao DEXA. Entretanto, os valores obtidos a partir das dobras cutâneas não foram significativamente diferentes daqueles obtidos pela DEXA. Florindo e cols. (54), avaliando pacientes com HIV/AIDS, verificaram que o percentual de gordura corporal medido pelo DEXA apresentou correlação com a gordura subcutânea total, central e de membros medidas pelo método de dobras cutâneas. Kamimura e cols. (55) observaram, em pacientes em hemodiálise há longo prazo, que os resultados não apresentaram diferenças entre as medidas de gordura corporal quando compararam os métodos de dobras cutâneas, BIA e DEXA.

É importante lembrar que nosso estudo tem como principal limitação o uso de uma técnica indireta para estimar o percentual de gordura corporal. $\mathrm{O}$ fato de não ter sido utilizada uma técnica considerada padrão de referência pode ter interferido na validade dos nossos resultados. No entanto, outros estudos já demonstram a validade tanto da técnica antropométrica por dobras cutâneas (37-42,71) como da técnica de BIA (43,7376) em comparação com o DEXA.

Considerando a limitação do estudo, destacamos sua importância pelo fato de não haver na literatura estudos feitos no país com esse tipo de metodologia, envolvendo amostras de indivíduos diabéticos tipo l e comparando a classificação do estado nutricional pelo método de IMC com três diferentes métodos de avaliação do percentual de gordura corporal, como os utilizados no presente estudo.

Outro aspecto de relevante importância em pacientes com DMl que justifica o correto diagnóstico do sobrepeso ou obesidade é que a intensificação do tratamento insulínico está resultando em uma melhora do controle clínico e metabólico da doença, com consequente aumento da sobrevida dos pacientes $(77,78)$. Porém, esse fato, quando associado ao aumento da prevalência de sobrepeso e/ou obesidade com aumento da gordura visceral, dislipidemia (aumento de triglicerídios, LDL-colesterol e ApoB) e aumento dos níveis pressão arterial nesses pacientes, poderia contribuir para um maior risco de desenvolvimento de complicações macrovasculares como, por exemplo, a doença arterial coronariana (79-81).

\section{CONCLUSÕES}

A utilização do IMC como método de classificação do estado nutricional mostrou-se inadequada, como já descrito anteriormente na literatura. No grupo de indivíduos diabéticos tipo 1 avaliados, tal método mostrouse pouco sensível às variações na composição corporal.

Embora os métodos de avaliação da composição corporal utilizados neste estudo (dobras cutâneas, BIA bipolar e tetrapolar) sejam validados a partir do padrão de referência que é a pesagem hidrostática ou DEXA, estudos comparativos devem ser realizados em indivíduos com DMl a fim de se afirmar qual deles apresenta resultados mais próximos aos observados no padrão-ouro, podendo, com isso, sugerir a substituição de um método pelo outro.

Considerando que a condição de sobrepeso ou obesidade está relacionada às complicações micro e macrovasculares do DM, a classificação do estado nutricional de indivíduos com DMl deve ser obtida por meio de métodos mais específicos de avaliação da composição corporal, nos quais a análise compartimentalizada do peso corporal seja realizada, visando, assim, à criação de intervenções clínicas terapêuticas que auxiliem na prevenção de tais complicações.

Declaração: os autores declaram não haver conflitos de interesse científico neste artigo.

\section{REFERÊNCIAS}

1. Wild S, Roglic G, Green A, Sicree R, King H. Global prevalence of diabetes. Estimates for the year 2000 and projections for 2030. Diabetes Care. 2004;27(5):1047-53. 
2. World Health Organization (WHO). Obesity: preventing and managing the global epidemic. Report of a WHO Consultation. WHO Technical Report Series 894. Geneva; 2000.

3. World Health Organization (WHO). Disponível em: <http://www.who. int/diabetes/facts/world_figures/en/>. Acesso em: 18 mar. 2008.

4. World Health Organization (WHO). Disponível em: <http://www.who. int/mediacentre/factsheets/fs311/en/>. Acesso em: 18 mar. 2008.

5. Fernqvist E, Gunnarsson R, Linde B. Influence of circulating epinephrine on absorption of subcutaneously injected insulin. Diabetes. 1988;37(6):694-701.

6. Landin-Olsson M, Karlsson FA, Lernmark A, Sundkvist G. Islet cell and thyrogastric antibodies in 633 consecutive 15- to 34-yrold patients in the diabetes incidence study in Sweden. Diabetes. 1992;41(8):1022-7.

7. Carneiro JRI, Kushnir MC, Clemente ELS, Brandão MG, Gomes MB. Obesidade na adolescência: fator de risco para complicações clínico-metabólicas. Arq Bras Endocrinol Metabol. 2000;44(5):390-6.

8. Invitti C, Guzzaloni G, Gilardini L, Morabito F, Viberti G. Prevalence and concomitants of glucose intolerance in European obese children and adolescents. Diabetes Care. 2003;26(1):118-24.

9. Moraes CM, Portella RB, Pinheiro VS, Oliveira MMS, Fuks AG, Cunha EF, et al. Prevalência de sobrepeso e obesidade em pacientes com diabetes tipo 1. Arq Bras Endocrinol Metabol. 2003;47(6):677-83.

10. Wilkin TJ. The accelerator hypothesis: weight gain as the missing link between type I and type II diabetes. Diabetologia. 2001;44(7):914-22.

11. Kibirige M, Metcalf $B$, Renuka R, Wilkin TJ. Testing the accelerator hypothesis: the relationship between body mass and age at diagnosis of type 1 diabetes. Diabetes Care. 2003;26(10):2865-70.

12. The Diabetes Control and Complications Trial Research Group (DCCT). Influence of intensive diabetes treatment on body weight and composition of adults with type 1 diabetes in the Diabetes Control and Complications Trial. Diabetes Care. 2001;24(10):1711-21.

13. Arcanjo CL, Piccirillo LJ, Machado IV, Andrade Jr CRM, Clemente EL, Gomes MB. Avaliação de dislipidemias e índices antropométricos em pacientes com diabetes mellitus tipo 1. Arq Bras Endocrinol Metabol. 2005;49(6):951-8.

14. Haney EM, Huffman LH, Bougatsos C, Freeman M, Steiner RD, Nelson HD. Screening and treatment for lipid disorders in children and adolescents: systematic evidence review for the US Preventive Services Task Force. Pediatrics. 2007;120(1):189-207.

15. World Health Organization (WHO). Physical status: the use and interpretation of anthropometry. Report of a WHO Expert Committee. WHOTechnical Report Series 854. Geneva; 1995.

16. Keys A, Fidanza F, Karvonen MJ, Kimura N, Taylor HL. Indices of relative weight and obesity. J Chronic Dis. 1972;25(6):329-43.

17. Lee J, Kolonel LN, Hinds MW. Relative merits of the weight-corrected-for-height indices. Am J Clin Nutr. 1981;34(11):2521-9.

18. Micozzi MS, Albanes D, Jones DY, Chumlea WC. Correlations of body mass indices with weight, stature, and body composition in men and women in NHANES I and II. Am J Clin Nutr 1986;44(6):725-31.

19. Norgan NG. Body mass index and body energy stores in developing countries. Eur J Clin Nutr. 1990;44(Supl1):79-84.

20. Peixoto MRG, Benício MHD, Jardim PCBV. Validade do peso e da altura auto-referidos: o estudo de Goiânia. Rev Saúde Públ. 2006;40(6):1065-72.

21. Silveira EA, Araújo CL, Gigante DP, Barros AJD, Lima MS. Validação do peso e altura referidos para o diagnóstico do estado nutricional em uma população de adultos no sul do Brasil. Cad Saúde Públ. 2005;21(1):235-45.
22. Fonseca MJM, Faerstein E, Chor D, Lopes CS. Validade de peso e estatura informados e índice de massa corporal: estudo prósaúde. Rev Saúde Públ. 2004;38(3):392-8.

23. Spencer EA, Appleby PN, Davey GK, Key TJ. Validity of self-reported height and weight in 4808 EPICOxford participants. Public Health Nutr. 2002;5(4):561-5.

24. Hill A, Roberts J. Body mass index: a comparison between selfreported and measured height and weight. J Public Health Med. 1998;20:206-10.

25. Schmidt MI, Duncan BB, Tavares M, Polanczyk CA, Pellanda L, Zimmer PM. Validity of self-reported weight: a study of urban Brazilian adults. Rev Saúde Públ. 1993;27(4):271-6.

26. Ellis KJ. Selected body composition methods can be used in field studies. J Nutr. 2001;131(5):1589-95.

27. Kyle UG, Genton L, Pichard C. Body composition: what's new. Curr Opin Clin Nutr Metab Care. 2002;5(4):427-33.

28. Womersley J, Durnin JVGA. A comparison of the skinfold method with extent of "overweight" and various weight-height relationships in the assessment of obesity. Brit J Nutr. 1977;38(2):271-82.

29. Frankenfield DS, Rowe WA, Cooney, RN, Smith JS, Becker D. Limits of body mass index to detect obesity and predict body composition. Nutrition. 2001;17(1):26-30.

30. Ricardo DR, Araújo CGS. Índice de massa corporal: um questionamento baseado em evidências. Arq Bras Cardiol. 2002;79(1):61-9.

31. Taylor RW, Falorni A, Jones IE, Goulding A. Identifying adolescents with high percentage body fat: a comparison of BMI cutoffs using age and stage of pubertal development compared with BMI cutoffs using age alone. Eur J Clin Nutr. 2003;57(6):764-9.

32. Heymsfield SB, Wang Z. Human body composition: advances in models and methods. Annu Rev Nutr. 1997:17:527-58.

33. Hammond J, Rona RJ, Chinn S. Estimation in community surveys of total body fat of children using bioelectrical impedance or skinfold thickness measurements. Eur J Clin Nutr. 1994;48(3):164-71.

34. Visser M, Langlois J, Guralnik JM, Cauley JA, Kronmal RA, Robbins $J$, et al. High body fatness, but not low fat-free mass, predicts disability in older men and women: the Cardiovascular Health Study. Am J Clin Nutr. 1998;68(3):584-90.

35. Zhu S, Wang Z, Shen W, Heymsfield SB, Heshka S. Percentage body fat ranges associated with metabolic syndrome risk: results based on the third National Health and Nutrition Examination Survey (1988-1994). Am J Clin Nutr. 2003;78(2):228-35.

36. Lukaski HC. Methods for the assessment of human body composition: traditional and new. Am J Clin Nutr. 1987;46(4):537-56.

37. Sloan AW. Estimation of body fat in young men. J Appl Physiol. 1967;23(3):311-5.

38. Jackson AS, Pollock ML. Generalized equations for predicting body density of men. Br J Nutr. 1978;40(3):497-504.

39. Jackson, A. S., Pollock, M. L., Ward, A. Generalized equations for predicting body density of women. Med Sci Sports Exerc 1980;12(3):175-82

40. Thorland WG, Johnson GO, Tharp GD, Housh TJ, Cisar CJ. Estimation of body density in adolescent athletes. Hum Biol. 1984;56(3):439-48.

41. Salem M, Fernandes Filho J, Pires Neto CS. Desenvolvimento e validação de equações antropométricas específicas para a determinação da densidade corporal de mulheres militares do Exército Brasileiro. Rev Bras Med Esporte. 2004;10(3):141-6.

42. Glaner MF, Rodriguez CRA. Validação de equações para estimar a densidade corporal e/ou percentual de gordura para militares masculinos. Trein Desportivo. 1999:4(3):29-36.

43. Pichard C, Kyle UG, Janssens JP, Burdet L, RochatT, Slosman DO, et al. Body composition by X-ray absorptiometry and bioelectrical impedance in chronic respiratory insufficiency patients. Nutrition. 1997;13(11-12):952-8. 
44. Kyle UG, Bosaeus I, De Lorenzo AD, Deurenberg P, Elia M, Gómez $\mathrm{JA}$, et al. Bioelectrical impedance analysis-part l: review of principles and methods. Clin Nutr. 2004;23(5):1226-43.

45. Kyle UG, Bosaeus I, De Lorenzo AD, Deurenberg P, Elia M, Gómez $\mathrm{JA}$, et al. Bioelectrical impedance analysis-part II: utilization in clinical practice. Clin Nutr. 2004;23(6):1430-53.

46. Pongchaiyakul C, Kosulwat V, Rojroongwasinkul N, Charoenkiatkul S, Thepsuthammarat K, Laopaiboon M, et al. Prediction of percentage body fat in rural thai population using simple anthropometric measurements. Obes Res. 2005;13(4):729-38.

47. Schoeller DA, Tylavsky FA, Baer DJ, Chumlea WC, Earthman CP, Fuerst T, et al. QDR 4500A dual-energy $\mathrm{X}$-ray absorptiometer underestimates fat mass in comparison with criterion methods in adults. Am J Clin Nutr. 2005;81(5):1018-125.

48. Glaner MF, Rosário WC. Validação cruzada de técnicas antropométricas para a estimativa da gordura corporal em homens. Revista Digital - Buenos Aires - Año 10 - № 82 - Marzo de 2005. Disponível em <http:// www.efdeportes.com/efd82/antrop.htm>. Acesso em: 5 abr. 2008.

49. Rodrigues MN, Silva SC, Monteiro WD, Farinatti PTV. Estimativa da gordura corporal através de equipamentos de bioimpedância, dobras cutâneas e pesagem hidrostática. Rev Bras Med Esporte. 2001;7(4):125-31.

50. Carvalho ABR, Pires Neto CS. Composição corporal através dos métodos da pesagem hidrostática e impedância bioelétrica em universitários. Revista Brasileira de Cineantropometria \& Desempenho Humano. 1999;1(1):18-23.

51. Lean MEJ, HanTS, Deurenberg P. Predicting body composition by densitometry from simple anthropometric measurements. Am J Clin Nutr. 1996;63(1):4-14.

52. Smalley KJ, Knerr AN, Kendrick ZV, Colliver JA, Owen OE. Reassessment of body mass indices. Am J Clin Nutr. 1990:52(3):405-8.

53. Freitas Jr IS, Rupp SAP, Godoy I, Santos SMS, Campana AO. Análise comparativa de métodos de avaliação da composição corporal em homens sadios e em pacientes com doença pulmonar obstrutiva crônica: antropometria, impedância bioelétrica e absortiometria de raios-X de dupla energia. Arch Latinoam Nutr 2005;55. Disponível em: <http://www.alanrevista.org/ediciones/2005-2/ analise_comparativa_corporal_em_homens_sadios.asp >. Acesso em 29 fev. 2008.

54. Florindo A, Latorre MRDO, Santos ECM, Borelli A, Rocha MS, Segurado AAC. Validação de métodos de estimativa da gordura corporal em portadores do HIV/Aids. Rev Saúde Publ. 2004;38(4):643-9.

55. Kamimura MA, Avesani CM, Cendoroglo M, Canziani MEF, Draibe SA, Cuppari L. Comparison of skinfold thicknesses and bioelectrical impedance analysis with dual-energy $\mathrm{X}$-ray absorptiometry for the assessment of body fat in patients on long-term haemodialysis therapy. Nephrol Dial Transplant. 2003;18(1):101-5.

56. Andrade Jr CRM, Clemente EL, Gomes MB. Influência da gordura corporal em parâmetros de controle clínico e metabólico de pacientes com diabetes mellitus tipo 1. Arq Bras Endocrinol Metabol. 2004;48(6):885-9.

57. Särnblad S, Ingberg CM, Aman J, Schvarcz E. Body composition in young female adults with type 1 diabetes mellitus. A prospective case-control study. Diabet Med. 2007;24(7):728-34.

58. Kistorp CN, Svendsen OL. Body composition analysis by dual energy $\mathrm{X}$-ray absorptiometry in female diabetics differs between manufacturers. Eur J Clin Nutr. 1997;51(7):449-54.

59. Leiter LA, The Diabetes Control and Complications Trial Research Group (DCCT). Use of bioelectrical impedance analysis measurements in patients with diabetes. Am J Clin Nutr. 1996;64(Suppl):515S-8S.

60. Guedes DP, Guedes JERP. Controle do peso corporal: composição corporal, atividade física e nutrição. Londrina: Midiograf; 1998.

61. Heyward VH, Stolarczyk LM. Avaliação da composição corporal aplicada. São Paulo: Manole; 2000.
62. Gallagher D, Heymsfield SB, Heo M, Jebb SA, Murgatroyd PR, Sakamoto $Y$. Healthy percentage body fat ranges: an approach for developing guidelines based on body mass index. Am J Clin Nutr. 2000;72(3):694-701.

63. Cohen JA coefficient of agreement for nominal scales. Educ Psychol Meas. 1960;20(1):37-46.

64. Malina RM, Katzmarz PT. Validity of the body mass index as an indicator of the risk and presence of overweight in adolescents. Am J Clin Nutr. 1999;70(1 pt 2):131-6.

65. Anjos LA. Índice de massa corporal (massa corporal.estatura-2) como indicador do estado nutricional de adultos: revisão de literatura. Rev Saúde Públ. 1992;26(6):203-15.

66. Carrasco FN, Reyes ES, Rimler OS, Rios FC. Exactitud del índice de masa corporal en la predicción de la adiposidad medida por impedanciometría bioeléctrica. Arch Latinoam Nutr. 2004;54(3):208-86.

67. Graves MM, Adams TM. Differences in disease risk stratification based on BMI versus percent body fat values. Med Sci Sports Exerc. 2004;36(Suppl 5):S75.

68. Khongsdier R. BMI and morbidity in relation to body composition: a cross-sectional study of a rural community in North-East India. Br J Nutr. 2005;93(1):101-7.

69. Garn SM, Leonard WR, Hawthorne VM. Three limitations of the body mass index. Am J Clin Nutr. 1986;44(6):996-7.

70. McLaren DS. Three limitations of body mass index. Am J Clin Nutr. 1987;46(1):121.

71. Glaner MF. Índice de massa corporal como indicativo da gordura corporal comparado às dobras cutâneas. Rev Bras Med Esporte. 2005;11(4):243-6.

72. Batista Filho $M$, Rissin A. A transição nutricional no Brasil: tendências regionais e temporais. Cad Saúde Públ. 2003;19(Supl 1):S181-91.

73. Brown DD, McKenzie JE, Cullen RW, Lagally KM, Dennies KK. A comparison of body composition techniques to determine body fat percentages in high school wrestlers. Med Sci Sports Exerc. 2004;36(Supl 5):S73.

74. Newton Jr. RL, Alfonso A, York-Crowe E, Walden H, White MA, Ryan $D$, et al. Comparison of body composition methods in obese african-american women. Obesity. 2006;14(3):415-22.

75. Sun SS, Chumlea WC, Heymsfield SB, Lukaski HC, Schoeller D, Friedl $\mathrm{K}$, et al. Development of bioelectrical impedance analysis prediction equations for body composition with the use of a multicomponent model for use in epidemiologic surveys. Am J Clin Nutr. 2003;77(2):331-40.

76. Sampei MA, Novo NF, Juliano Y, Sigulem DM. Comparison of the body mass index to other methods of body fat evaluation in ethnic Japanese and Caucasian adolescent girls. Int $\mathrm{J}$ Obes. 2001;25(3):400-8.

77. Nishimura R, LaPorte RE, Dorman JS, Tajma N, Becker D, Orchard TJ, et al. Mortality trends in type 1 diabetes. Diabetes Care. 2001;24(5):823-7.

78. Schaumberg DA, Glynn RJ, Jenkins AJ, LyonsTJ, Rifai, N, Manson $\mathrm{JE}$, et al. Effect of intensive glycemic control on levels of markers of inflammation in type 1 diabetes mellitus in the Diabetes Control and Complications Trial. Circulation. 2005;111(19):2446-53.

79. Purnell JQ, Hakanson JE, Marcovina SM, Steffes MW, Cleary PA, Brunzell JD. Effect of excessive weight gain with intensive therapy of type 1 diabetes on lipid levels and blood pressure. JAMA. 1998;280(23):140-6.

80. Franz MJ, Bantle JP, Beebe CA, Brunzell JD, Chiasson JL, Garg A et al. Evidence-based nutrition principles and recommendations for the treatment and prevention of diabetes and related complications. Diabetes Care. 2002;25(10):148-98.

81. Sibley SD, Palmer, JP, Hirsch IB, Brunzell JD. Visceral obesity, hepatic lipase activity, and dyslipidemia in type 1 diabetes. J Clin Endocrinol Metab. 2003;88(7):3379-84. 